
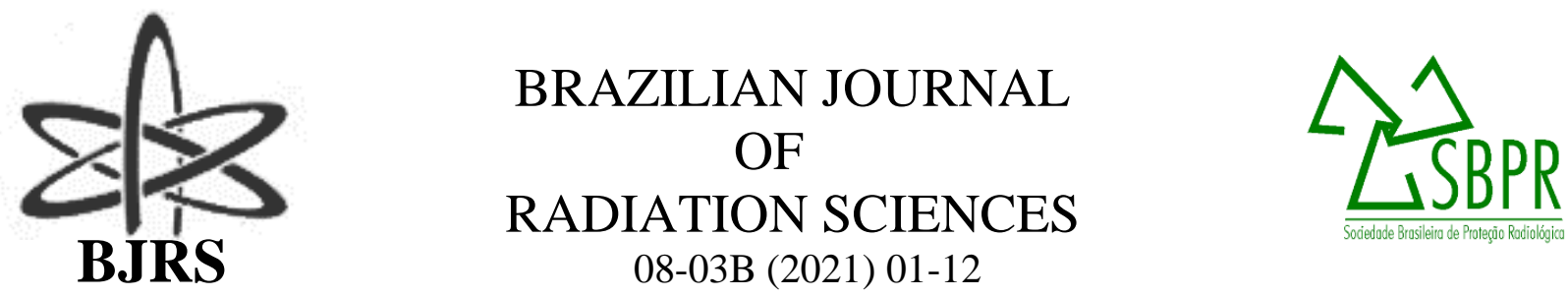

\title{
Curvilinear coordinate system used to solve tritium dispersion in complex orographies
}

\author{
Meneghetti $^{\mathrm{a}, \mathrm{b}}$ A., Bodmann ${ }^{\mathrm{b}}$ B.E.J., Vilhena ${ }^{\mathrm{b}}$ M.T.M.B. \\ ${ }^{a}$ IMEF/FURG, 96203-900, Rio Grande, Rio Grande do Sul, Brasil \\ ${ }^{b}$ PROMEC/UFRGS, 90050-170, Porto Alegre, Rio Grande do Sul, Brasil \\ andremeneghetti@furg.br
}

\begin{abstract}
In this contribution we present progress on research concerning dispersion of tritium around the Angra Nuclear Power Plant (Angra dos Reis, Rio de Janeiro state, Brazil). In particular, we are interested in studying how dispersion behaves in scenarios with complex orography. Our proposal is to transform a problem with curvilinear boundaries into an equivalent problem with plane parallel boundaries. We modify the coordinate system through a diffeomorph conformal transformation. Consequently, the operators of the dynamical equations change according to the additional terms from the affine connection. To define the transformation it is necessary to satisfy strong constraints, i.e., boundaries shall be "smooth". Even with restrictions, our model can be implemented in several situations. A flat region is a particular case of a curvilinear domain and can be studied, where the height of the boundary layer above rivers, lakes, basins is typically smaller and thus implies a varying boundary layer height, for example. Thus, even in flat regions variations in the boundary layer occur, which characterizes a case of a curvilinear domain. There are several nuclear power plants worldwide, that are located in mountainous regions, as for example in Japan and Brazil. We present a simulation of tritium dispersion specifically in the area where the Angra 2 Nuclear Power Plant is located and where the relief is characterized by a considerable complexity. With the intention of showing that the methodology is consistent, we will consider only the variation of the relief.
\end{abstract}

Keywords: tritium, dispersion, diffeomorph, conformal, transformation. 


\section{INTRODUCTION}

The objective of our research is to study flow problems in domains with curvilinear boundaries. The proposal consists of modifying the coordinate system. To this end, we construct a function $T: \Omega \subseteq \mathbb{R}^{3} \rightarrow \mathbb{R}^{3}$ that transforms the domain $\Omega$, our curvilinear domain, into a domain whose boundaries are plane-parallel. The transformation of the coordinate system changes not only the domain but also the partial differential equation that models the problem. Unlike conventional methodologies, which do not change the domain, we propose to transfer the difficulty found in the non-planar boundaries to the differential equation. If we initially have traditional partial differential equations (such as the Navier-Stokes equations, continuity equation and advection-diffusion equation) that model a problem on a domain whose boundaries are curvilinear, after transformation we have transformed partial differential equations modeling the problem on a domain that has plane-parallel boundaries.

This proposal for solving problems of flows was motivated in particular by classical theories such as Differential Geometry and Tensorial Calculus. As we can see in [1,2], these are well developed theories and, under certain domain conditions guarantee that the proposed methodology is valid. Once transformed, the partial differential equation can be solved by known methods.

In this work, supported mainly by [2,3], we use numerical resolution techniques. Coordinate transformation together with numerical resolution methods allow for robust modeling that can be applied to multiple scenarios. An application of relevance for the nuclear community, see references [4-6], is to calculate the tritium dispersion around the region of Angra dos Reis, Rio de Janeiro, Brazil. In this paper we describe part of this study. Although, some simplifications were used, the methodology proved effective, as can be seen in the following sections.

\section{TRANSFORMATION OF THE COORDINATE SYSTEM}

Let $x^{1} \times x^{2} \times x^{3}$ be the Cartesian coordinate system and $\xi^{1} \times \xi^{2} \times \xi^{3}$ the generalized coordinate system. We define the general transformation $T$ by conformal equation (1). 


$$
T: \xi^{\alpha}=\xi^{\alpha}\left(x^{i}\right)
$$

In equation (1), $i, \alpha \in\{1,2,3\}, x^{i}=\left\{x^{1}, x^{2}, x^{3}\right\} \in \Omega \subseteq \mathbb{R}^{3}$, where $\Omega$ is an open set. The transformation $\mathrm{T}$ must satisfy the property of being a diffeomorph conformal transformation. In addition to the mathematical characteristics, this property ensures that conservation laws are preserved. According to [7], $T$ is a conformal diffeomorph transformation if and only if the functions $\xi^{\alpha}$ are of class $C^{1}(\Omega)$ and $|J| \neq 0$ in $\Omega$, where $J$ is the Jacobian determinant of transformation T. See equation (2).

$$
J=\left|\frac{\partial \xi^{\alpha}}{\partial x^{i}}\right| \neq 0
$$

While $x^{1}, x^{2}, x^{3}$ are generic, the transformation $T$ transforms the system of Cartesian coordinates into the generalized coordinate system. After defining the functions $x^{1}, x^{2}, x^{3}$, we say that $T$ transforms the Cartesian coordinate system into a curvilinear coordinate system.

\section{THE ADVECTION-DIFFUSION EQUATION}

A dispersion problem can be modeled by the advection-diffusion equation. Using the simplified equation and Einstein summation convention, with $i \in\{1,2,3\}$, we obtain the equation (3).

$$
\frac{\partial C}{\partial t}+u^{i} \frac{\partial C}{\partial x^{i}}=\frac{\partial K_{x^{i}}}{\partial x^{i}} \frac{\partial C}{\partial x^{i}}+K_{x^{i}} \frac{\partial^{2} C}{\partial\left(x^{i}\right)^{2}}
$$

Here, the function $C$ is the average concentration of a contaminant $\left[\mathrm{g} / \mathrm{m}^{3}\right]$. The scalar functions $u^{i}$ are the wind speeds $[\mathrm{m} / \mathrm{s}]$ in the direction of the $x^{1}, x^{2}, x^{3}$ axes of the Cartesian coordinate system, respectively. The coefficients $K_{x^{1}}, K_{x^{2}}, K_{x^{3}}$ are the turbulent diffusion coefficients $\left[\mathrm{m}^{2} / \mathrm{s}\right]$, respectively, in the directions $x^{1}, x^{2}, x^{3}$. 


\section{THE TRANSFORMED ADVECTION-DIFFUSION EQUATION}

The variables of both coordinate systems are connected using the chain rule. Equations (4) and (5) show the first derivative already transformed the functions $C$ and $K_{x^{i}}$.

$$
\begin{gathered}
\frac{\partial C}{\partial x^{i}}=\frac{\partial C}{\partial \xi^{\alpha}} \frac{\partial \xi^{\alpha}}{\partial x^{i}} \\
\frac{\partial K_{x^{i}}}{\partial x^{i}}=\frac{\partial K_{x^{i}}}{\partial \xi^{\alpha}} \frac{\partial \xi^{\alpha}}{\partial x^{i}}
\end{gathered}
$$

In these equations, $i, \alpha \in\{1,2,3\}$. The second derivatives, in the transformed form, are described by equation (6), where $i, j, \alpha, \beta \in\{1,2,3\}$ and the Kronecker symbol $\delta_{j}^{i}=1$ for $i=j$ and zero otherwise.

$$
\frac{\partial^{2} C}{\partial\left(x^{i}\right)^{2}}=\delta_{j}^{i} \frac{\partial C}{\partial \xi^{\alpha}} \frac{\partial \xi^{\alpha}}{\partial x^{i}}+\frac{\partial^{2} C}{\partial \xi^{\alpha} \partial \xi^{\beta}} \frac{\partial \xi^{\alpha}}{\partial x^{i}} \frac{\partial \xi^{\beta}}{\partial x^{i}}
$$

Replacing (4), (5) and (6) in (3), we obtain the advection-diffusion equation in generalized coordinates, conformal equation (7).

$$
\frac{\partial C}{\partial t}+u^{i} \frac{\partial C}{\partial \xi^{\alpha}} \frac{\partial \xi^{\alpha}}{\partial x^{i}}=\frac{\partial K_{x^{i}}}{\partial \xi^{\alpha}} \frac{\partial \xi^{\alpha}}{\partial x^{i}} \frac{\partial C}{\partial \xi^{\beta}} \frac{\partial \xi^{\beta}}{\partial x^{i}}+K_{x^{i}}\left(\delta_{j}^{i} \frac{\partial C}{\partial \xi^{\alpha}} \frac{\partial \xi^{\alpha}}{\partial x^{i}}+\frac{\partial^{2} C}{\partial \xi^{\alpha} \partial \xi^{\beta}} \frac{\partial \xi^{\alpha}}{\partial x^{i}} \frac{\partial \xi^{\beta}}{\partial x^{i}}\right)
$$

\section{NUMERICAL RESOLUTION}

We solve a problem that has velocities $u^{i}$ and diffusion coefficients $K_{x^{i}}$ varying only in space. The solution of equation (7) can be estimated using a numerical resolution. In the present approach we use the finite difference method. Before starting, we simplify the equation. The coefficients in $C$ are put in evidence, thus, we obtain the equation (8). 


$$
\begin{gathered}
\frac{\partial C}{\partial t}=D_{1} \frac{\partial C}{\partial \xi^{1}}+D_{2} \frac{\partial C}{\partial \xi^{2}}+D_{3} \frac{\partial C}{\partial \xi^{3}}+D_{4} \frac{\partial C}{\partial\left(\xi^{1}\right)^{2}}+D_{5} \frac{\partial C}{\partial\left(\xi^{2}\right)^{2}}+D_{6} \frac{\partial C}{\partial\left(\xi^{3}\right)^{2}}+ \\
D_{7} \frac{\partial^{2} C}{\partial \xi^{1} \partial \xi^{2}}+D_{8} \frac{\partial^{2} C}{\partial \xi^{1} \partial \xi^{3}}+D_{9} \frac{\partial^{2} C}{\partial \xi^{2} \partial \xi^{3}}
\end{gathered}
$$

We highlight the dependence of the coefficients $D_{i}=D_{i}\left(x^{1}, x^{2}, x^{3}, K_{x^{1}}, K_{x^{2}}, K_{x^{3}}, u^{1}, u^{2}, u^{3}\right)$, where $i \in\{1,2, \ldots, 9\}$. The derivative with respect to time is discretized using ascending finite differences, while the derivatives with respect to space are discretized using central finite differences. Thus, equation (8) can be approximated by a matrix equation (9).

$$
E \vec{C}_{n+1}=\vec{C}_{n}+\vec{B}
$$

Let $N \xi^{i} \in \mathbb{N}$ where $N \xi^{i}+2$ is the number of nodes with respect to the axis $\xi^{i}$, for $i \in\{1,2,3\}$. In equation (9), $E$ is a matrix of dimension $\left(N \xi^{1} N \xi^{2} N \xi^{3}\right) \times\left(N \xi^{1} N \xi^{2} N \xi^{3}\right)$. The elements $\vec{C}_{n+1}, \vec{C}_{n}$ and $\vec{B}$ are vectors of dimension $\left(N \xi^{1} N \xi^{2} N \xi^{3}\right) \times 1$.

The matrix $E$ is formed by the dynamic coefficients, all evaluated at time $n$. Therefore, the matrix $E$ is known. The vector $\vec{C}_{n}=\left[C_{i, j, l, n}\right]_{i, j, l}$ is also known. The vector $\vec{B}$ is formed by values obtained at the boundaries and evaluated at time $n+1$, whereas the vector $\vec{C}_{n+1}=\left[C_{i, j, l, n+1}\right]_{i, j, l}$ is to be determined for each iteration step. To solve the matrix equation (9) we use an approximation for the vector $\vec{B}$, more precisely, we use $\vec{B}$ at time $n$ which is known.

\section{RELIEF APPROACH FROM DATA TAKEN FROM GOOGLE EARTH}

The domain that will be used for the construction of the mesh has parallel planes in its boundaries, except the lower boundary. For the inferior boundary we construct a function that approximates the relief located in the region of interest, which is the region around the Angra 2 nuclear power plant. 
Google-Earth was used to obtain an approximation of the surface around of Angra 2 power plant. The region of interest was covered with a grid in the more frequent direction of wind. Data on wind speed and direction frequency were obtained from [8]. With this grid it was possible to obtain strategic points with information about relative height. This information was obtained using the "elevation profile" feature available on Google-Earth. The Figure 1, obtained by Google Earth, illustrates the region of Angra 2 power plant. In the center of the circle, a chimney of approximately $160 \mathrm{~m}$ high releases effluents. The red line shows one of the most frequent direction of the wind.

Figure 1: Illustration of the Angra 2 region.

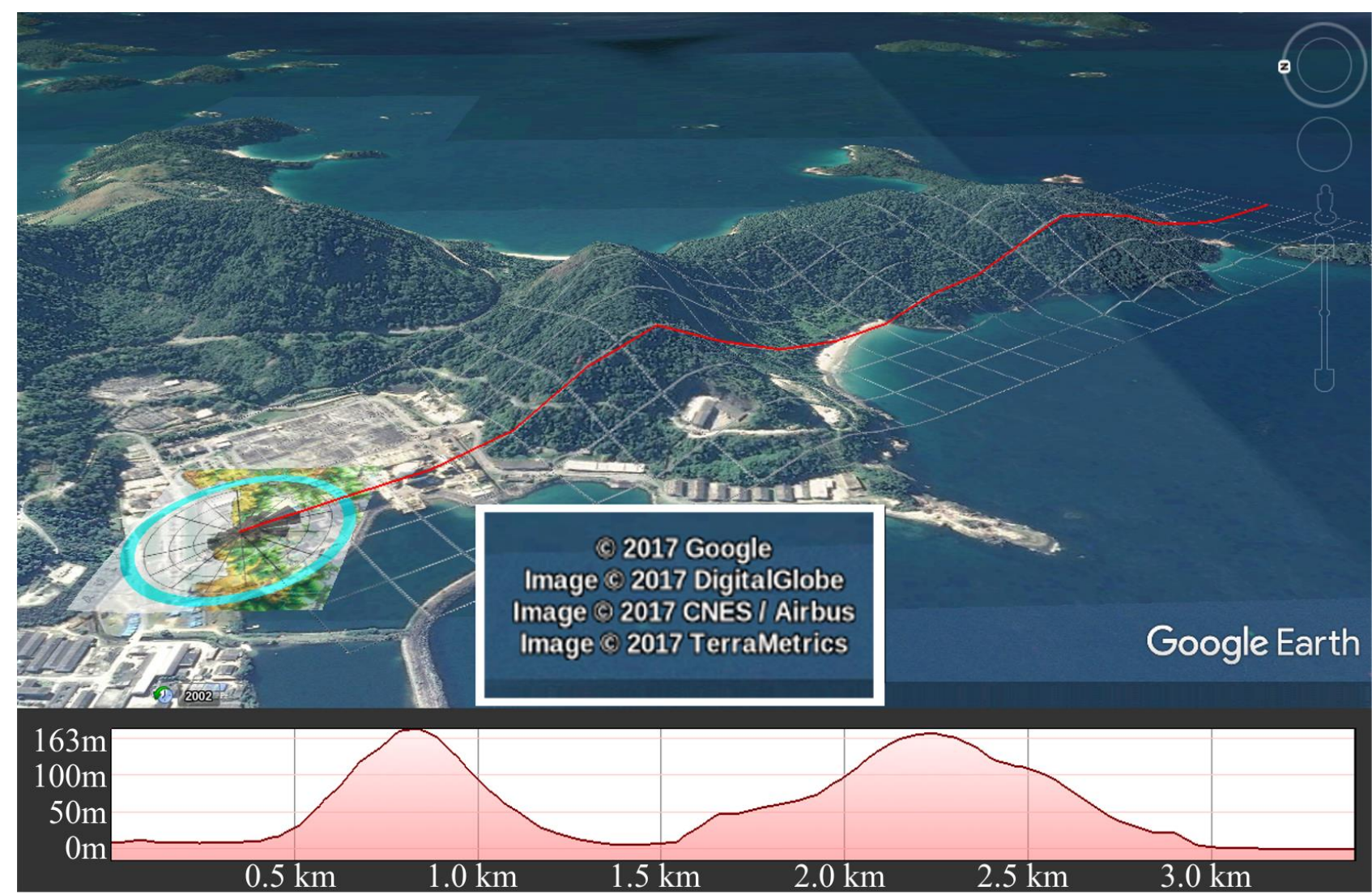

Source: Google Earth Linux version

The red dots in Figure 2 were obtained by Google-Earth. Using interpolation we obtain a surface that approximates the region of interest. 
Figure 2: Interpolation around the Angra 2 power plant region.

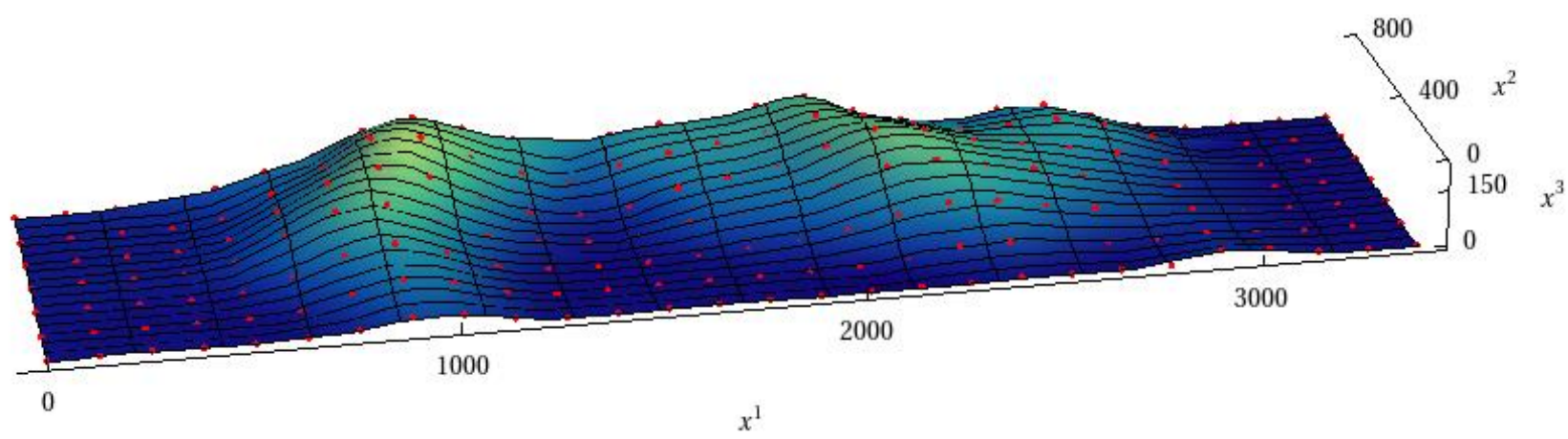

Interpolation provides the function that approximates the region. We use this function to define the nodes we need. Once the lower mesh is obtained, we define the upper mesh. The upper mesh is rectangular and thus does not cause difficulties in its definition. From the lower and upper meshes, we define the inner nodes.

\section{SIMULATION}

Our methodology was tested using a region around the Angra 2 nuclear power plant. The physically relevant domain resides in a volume of a parallelepiped with $x \in[0,3400 m], y \in$ $[0,800 \mathrm{~m}]$ and $z \in[0,800 \mathrm{~m}]$. The chimney output is identified by the coordinates $(0,400 \mathrm{~m}, 160 \mathrm{~m})$.

The choice of the domain dimensions is related to data comparison and the parametrisation used for the diffusion coefficients. In micro-meteorology these properties refer to domains that have approximately the same dimensions. We solved equation (3) with the following initial and boundaries conditions, equation (10).

Here $z_{i}$ is the height, in meters, of the relief relative to sea level at the coordinate point $\left(x^{1}, x^{2}\right)$, $D=8 \times 10^{-8} \mathrm{~g} / \mathrm{m}^{3} \delta\left(x^{2}-400\right) \delta\left(x^{3}-160\right)$ where $\delta$ is the Dirac's delta function. The wind speed as well as the diffusive coefficients was defined as constants. We considered $u=3.8 \mathrm{~m} / \mathrm{s}$, $v=0, w=0, K_{x^{1}}=10 \mathrm{~m}^{2} / \mathrm{s}, K_{x^{2}}=10 \mathrm{~m}^{2} / \mathrm{s}, K_{x^{3}}=10 \mathrm{~m}^{2} / \mathrm{s}$. 


$$
\begin{array}{cll}
C\left(x^{1}, x^{2}, x^{3}, 0\right)=0, & \forall x^{1} \in[0,3400 m], \forall x^{2} \in[0,800 m] \text { and } \forall x^{3} \in\left[z_{i}, 800 m\right] \\
\left.\frac{\partial C}{\partial x^{2}}\right|_{x^{2}=0}=0, & \forall x^{1} \in[0,3400 m], \forall x^{3} \in\left[z_{i}, 800 m\right] \text { and } \forall t \in[0, \infty) ; \\
\left.\frac{\partial C}{\partial x^{2}}\right|_{x^{2}=800 m}=0, & \forall x^{1} \in[0,3400 m], \forall x^{3} \in\left[z_{i}, 800 m\right] \text { and } \forall t \in[0, \infty) ; \\
\left.\frac{\partial C}{\partial x^{2}}\right|_{x^{3}=z_{i}}=0, & \forall x^{1} \in[0,3400 m], \forall x^{2} \in[0,800 m] \text { and } \forall t \in[0, \infty) ; \\
\left.\frac{\partial C}{\partial x^{2}}\right|_{x^{3}=z_{i}}=0, & \forall x^{1} \in[0,3400 m], \forall x^{2} \in[0,800 m] \text { and } \forall t \in[0, \infty) ; \\
\left.\frac{\partial C}{\partial x^{2}}\right|_{x^{1}=0}=0, & \forall x^{2} \in[0,800 m] \text { and } \forall x^{3} \in\left[z_{i}, 800 m\right] \text { and } \forall t \in[0, \infty) ; \\
\left.C\right|_{x^{1}=0}=D, & \forall x^{2} \in[0,800 m] \text { and } \forall x^{3} \in\left[z_{i}, 800 m\right] \text { and } \forall t \in[0, \infty) .
\end{array}
$$

Note, that Gaussian models are the simplest ones and usually use constant diffusive coefficients. In this application, 131 partitions were used in the direction of the $x^{1}$-axis, 31 partitions in the $x^{2}$-axis direction and 31 partitions in the $x^{3}$-axis direction.

\subsection{RESULTS}

To illustrate the results we present the Figure 3 and Figure 4. The Figure 2 shows the region of Angra dos Reis that was parametrised. We highlight the slice that contains the chimney that releases the effluent. In Figure 4, the same slice is shown as profile. The concentration of tritium released as effluent was determined at each node of the mesh, in which we plotted on a scale from 0 to $10^{-9} \mathrm{~g} / \mathrm{m}^{3}$.

We use the increment in time being $\Delta t=0.1 \mathrm{~s}$. To obtain that maximum error between two iterations was less than $10^{-6} \mathrm{~g} / \mathrm{m}^{3}, 9600$ iterations were required. This represents approximately 16 minutes of simulation in a desktop computer with a simple quad core processor with $3.2 \mathrm{GHz}$ clock. The time taken to generate the result was 11 minutes approximately. We used the $\mathrm{C}$ programming language.

Figure 3: Tritium dispersion for $x^{2}=400 m, x^{1} \in(, 3400 m)$ and $x^{3} \in\left(z_{i}, 800 m\right)$. 


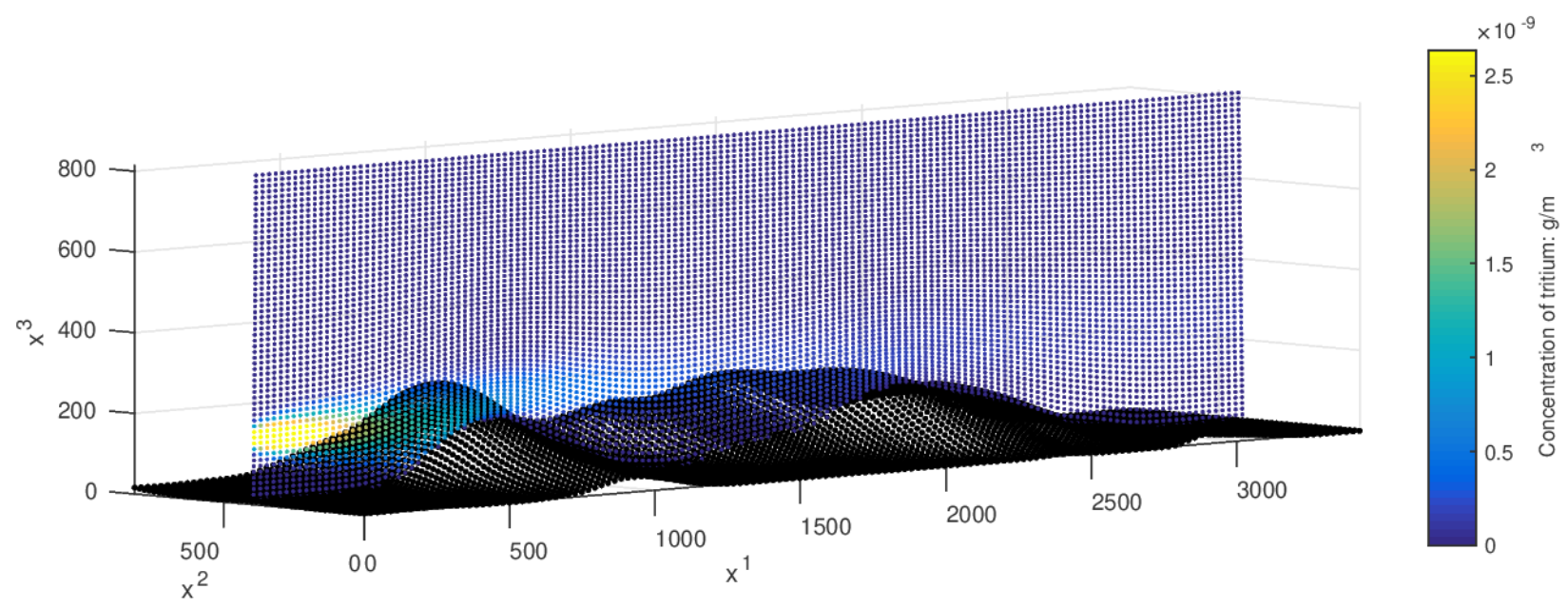

Figure 4: Profile of Figure 3.

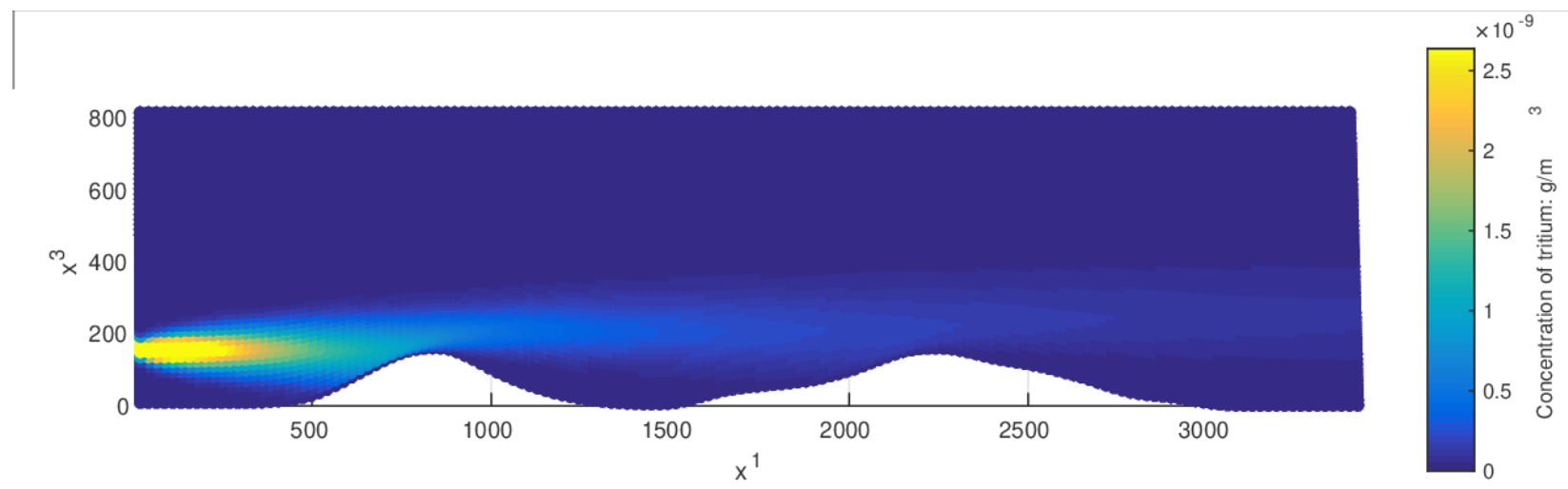

\subsection{ERROR - RESIDUE}

In order to verify the consistency of the obtained solution we use the residue technique. From the advection-diffusion equation, equation (3), we define the operator (11).

$$
\Omega\left(u^{i}\right)=\frac{\partial C}{\partial t}+u^{i} \frac{\partial C}{\partial x^{i}}-\frac{\partial K_{x^{i}}}{\partial x^{i}} \frac{\partial C}{\partial x^{i}}-K_{x^{i}} \frac{\partial^{2} C}{\partial\left(x^{i}\right)^{2}}
$$

We use the solution $C$ in the operator (11). In replacing, we expect to find as zero solution or, more precisely, a solution very close to zero which is known in classical theory as residue. 
If we call $n+1$ the last iteration performed, then we can say that $n+1$ and $n$ iterations will be used. This will be necessary, since in order to test the obtained solution we will again use the implicit finite difference method. The operator (11) can be approximated using the implicit finite difference method. We present the same plane $\left(x^{1}, 400 m, x^{3}\right)$. To see the error it is necessary to reduce the order of magnitude, as shown in Figure 5.

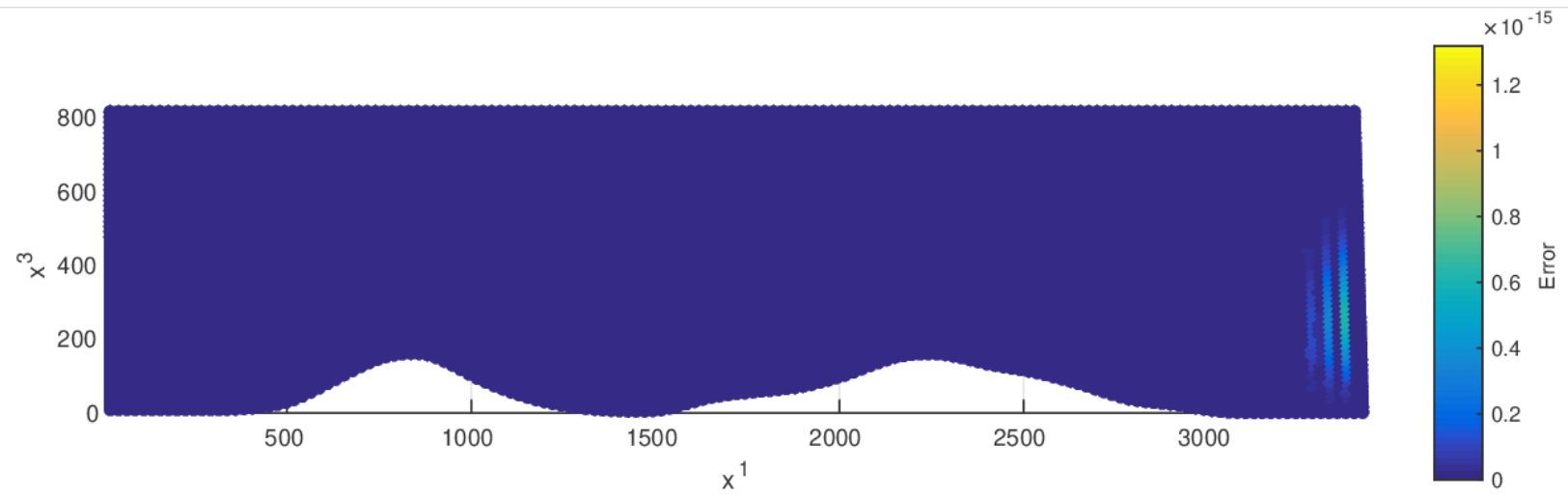

Figure 5: Residue of the region

\section{CONCLUSION}

This work shows an application where a dispersion process in an environment with complex orography was simulated. Here we implemented tritium dispersion around the Angra dos Reis nuclear power plant. This plant is located in a mountainous region, which may invalidate dispersion models

that are developed to work in parallel flat domains. We present an efficient way to parametrise mountain regions using basically Google-Earth software. In addition, we show the main idea about the methodology needed to transform a problem that has a domain with curvilinear boundaries in an equivalent problem with parallel plane boundaries by a diffeomorph conformal coordinate transformation. The authors are aware of the fact that there are many problems that cannot be solved using this technique, nevertheless there is still a wide range of applications. To this end the original problem was transformed into a plane parallel problem by the use of the afore mentioned transformation, 
which are well established formalisms in differential geometry. Another point to highlight is that such types of coordinate transformations preserve conservation laws. The presented results are in agreement with expectations presented in the literature, reference [5], but will be further validated against experimental data from a future campaign which is still to be implemented. In order to make further progress, we have two proposals for the continuation of our research on this topic. The first one will be to improve the numerical resolution, introducing techniques that will make the code more robust and consequently we will obtain more precise results.

The other proposal, already under development, is to present a semi-analytical solution. Numerical solutions accept more complex models, but with high computational cost and often time consuming results. On the other hand, currently, semi-analytical solutions solve more simplified models, however with the advantage of fast answers and with low computational cost. While there is no miraculous universal solver method, we will analyze and compare the relevance and effectiveness of both cited resolution techniques. 


\section{ACKNOWLEDGMENT}

The authors would like to thank CNPq for financial support.

\section{REFERENCES}

[1] CARMO , M.P. Differential geometry of curves and surfaces, Dover Publications Inc, 2017.

[2] SOKOLNIKOFF, I. S. Tensor Analysis, John Wiley \& Sons, Inc, New York \& USA, 1962.

[3] HOFFMAN, J. D. Numerical Methods for Engineers and Scientists, McGraw-Hill Book Company, New York \& USA, 1992.

[4] MOREIRA, D. M. ; VILHENA, M. T. ; TIRABASSI, T. ; CARVALHO, J. C. A semianalytical model for the Tritium dispersion simulation in the PBL from the Angra I nuclear power plant, Ecological Modelling, v. 189, n.3-4, p. 413-424, 2005.

[5] MOREIRA, D. M. ; VILHENA, M. T. ; CARVALHO, J. C. Tritium dispersion simulation in the atmosphere from ANGRA I Nuclear Power Plant, International Journal of Nuclear Energy, Science and Technology (Print), v. 3, p. 118-130, 2007.

[6] WEYMAR, G.J.; BUSKE, D.; VILHENA, M.T.M.B.; BODMANN, B.E.J. Validating a Closed Form AdvectionDiffusion Solution by Experiments: Tritium Dispersion after Emission from the Brazilian Angra Dos Reis Nuclear Power Plant, In: Integral Methods in Science and Engineering: Progress in Numerical and Analytic Techniques. Birkhuser/Springer, New York, 2013, p. 385.

[7] MENEGHETTI, A.; BODMANN, B.E.J. A.; VILHENA, M.T.M.B. New Diffeomorph Conformal Methodology to Solve Flow Problems with Complex Boundaries by an Equivalent Plane Parallel Problem, In: Integral Methods in Science and Engineering, Volume 1 Birkhuser/Springer, New York, 2017. p. 205-214.

[8] Estado do Rio de Janeiro - Atlas Elico, Available at: <http://www.cresesb.cepel.br/publicacoes/download/atlas_eolico/AtlasEolicoRJ.pdf> Last accessed: 10 Sept. 2017. 